\title{
Downregulation of LINC01638 IncRNA inhibits migration and invasion of pancreatic ductal adenocarcinoma cells by reducing TGF- $\beta$ signaling
}

\author{
HUIMIN LU, JUN YE, LING ZHANG, MAO LI, SHAN LU, DUJIANG YANG and WEIMING HU \\ Department of Pancreatic Surgery, West China Hospital, Sichuan University, Chengdu, Sichuan 610041, P.R. China
}

Received July 28, 2018; Accepted February 22, 2019

DOI: $10.3892 / \mathrm{mmr} .2019 .10699$

\begin{abstract}
LINC01638 is a long non-coding RNA (lncRNA) with an oncogenic role in breast cancer, while its involvement in other malignancies is unknown. This study was performed to investigate the potential role of LINC01638 in pancreatic ductal adenocarcinoma (PDAC). The expression of LINC01638 was determined via reverse transcription-quantitative polymerase chain reaction analysis, whereas the levels of transforming growth factor- $\beta 1$ (TGF- $\beta 1$ ) in plasma were measured via ELISA. Receiver operating characteristic curve analysis was conducted to determine the diagnostic value of LINC01638. Additionally, the migratory and invasive abilities of cells were evaluated via Transwell migration and invasion assays. In the present study, LINC01638 was significantly upregulated in tumor tissues compared with adjacent healthy tissues in the majority of patients with PDAC. Plasma levels of LINC01638 were significantly higher in patients with PDAC compared with in healthy controls. In effect, upregulation of plasma LINC01638 distinguished patients with PDAC from healthy controls in receiver operating characteristic analysis. Plasma levels of LINC01638 and TGF- $\beta 1$ were positively correlated in patients with PDAC, but not in healthy controls. LINC01638 overexpression increased TGF- $\beta 1$ expression, while silencing of LINC01638 using short hairpin RNA (shRNA) led to reduced TGF- $\beta 1$ expression in a PDAC cell line. LINC01638 overexpression promoted, while shRNA silencing inhibited, migration and invasion of cell of a PDAC cell line. Treatment with exogenous TGF- $\beta 1$ attenuated the inhibitory effect of LINC01638 shRNA silencing on cancer cell migration and invasion. It is concluded that LINC01638 lncRNA may be involved in the migration and invasion of PDAC cells via regulation of TGF- $\beta 1$.
\end{abstract}

Correspondence to: Dr Weiming Hu, Department of Pancreatic Surgery, West China Hospital, Sichuan University, 37 Guoxue Xiang, Chengdu, Sichuan 610041, P.R. China

E-mail: weiminghu626@sina.com

Key words: pancreatic ductal adenocarcinoma, LINC01638 long non-coding RNA, transforming growth factor- $\beta 1$, metastasis, pancreatic cancer

\section{Introduction}

Cases of pancreatic cancer account for $3 \%$ of all new cancer diagnoses; however this disease the fourth leading cause of cancer-associated mortality due to its highly aggressive nature (1). With the increasing incidence of pancreatic cancer, this disease is predicted to be the second most common cause of cancer-associated mortality by 2030 (2). Pancreatic ductal adenocarcinoma (PDAC), which develops from the exocrine pancreas, is the most common type of pancreatic cancer and accounts for $95 \%$ of pancreatic cancer cases (3). Surgical resection is the only treatment that prolongs survival; however, the 5-year survival rates are low even after curative resection (4-6).

Transforming growth factor- $\beta$ (TGF- $\beta$ ) signaling has a central role in the development of multiple cancers, including PDAC $(7,8)$. The activation of TGF- $\beta$ signaling promotes epithelial-mesenchymal transition (EMT) to mediate metastasis of PDAC tumors (9), which is a major cause of failure in treatment and the high mortality rate among patients with PDAC. TGF- $\beta$ signaling is involved in the pathogenesis of PDAC through interactions with multiple regulatory molecules, such microRNAs (miRNAs) (10). Interactions between TGF- $\beta$ signaling and long non-coding RNAs (lncRNAs) in PDAC have not been well investigated. LINC01638 is a recently characterized lncRNA with an oncogenic role in triple breast cancer (11). LINC01638 IncRNA promotes the development and progression of triple-negative breast cancer by activating metadherin-Twist1 signaling through the inhibition of speckle-type $\mathrm{POZ}$ protein-mediated c-Myc degradation (11); however, the role of LINC01638 lncRNA in other diseases is unknown. A preliminary microarray analysis was performed and revealed that LINC01638 IncRNA was upregulated in PDAC, indicating the potential role of LINC01638 lncRNA in PDAC. The findings of the present study indicated that LINC01638 may upregulate TGF- $\beta$ to regulate the migration and invasion of PDAC cells.

\section{Materials and methods}

Patients and specimens. A total of 102 patients with PDAC were diagnosed and treated in West China Hospital (Chengdu, China) between March 2016 and March 2018. The current study included 46 of the 102 patients according to inclusion and 
exclusion criteria. Inclusion criteria were as follows: i) Patients were diagnosed with PDAC via biopsies; ii) patients were willing to donate adjacent healthy tissue biopsies; iii) patients fully understood the experimental protocol and were willing to participate. Exclusion criteria were as follows: i) Patients with other malignancies; ii) patients with chronic diseases; iii) patients that had received treatment for any disease within 3 months prior to sampling. The study also included 38 healthy volunteers from whom samples were collected at the aforementioned hospital during the same time period to serve as the control group. Controls were age- and sex-matched to the patient group. Volunteers with a family history of malignancy were excluded. No significant differences in age and sex were observed between the two groups. Basic information is presented in Table I. This study was approved by the ethics committee of West China Hospital. All participants signed informed consent and fully understood the experimental protocol.

Specimen collection. Blood $(5 \mathrm{ml})$ was extracted from each participant 1 day after admission before breakfast. Blood samples were used to prepare plasma. Briefly, blood was centrifuged at $1,200 \mathrm{x} \mathrm{g}$ in EDTA tubes at room temperature for $20 \mathrm{~min}$, and the supernatant (plasma) was collected. Tumor tissues and adjacent healthy tissues within $2 \mathrm{~cm}$ around tumors were collected from 3 different sites in each PDAC patient through fine needle biopsy. All samples were kept in liquid nitrogen prior to use.

Reverse transcrttpion-quantitative polymerase chain reaction. TRIzol ${ }^{\circledR}$ reagent (Invitrogen; Thermo Fisher Scientific, Inc., Waltham, MA, USA) was used to extract total RNA with all operations performed in strict accordance with manufactuer's instructions. cDNA was synthesized using SuperScript IV Reverse Transcriptase (Thermo Fisher Scientific, Inc.) under the following conditions: $25^{\circ} \mathrm{C}$ for $5 \mathrm{~min}, 55^{\circ} \mathrm{C}$ for $30 \mathrm{~min}$ and $75^{\circ} \mathrm{C}$ for $10 \mathrm{~min}$. SYBR ${ }^{\circledR}$ Green Real-Time PCR Master Mixes (Thermo Fisher Scientific, Inc.) was used to prepare all PCR rection systems. PCR reaction conditions were: 1 min at $95^{\circ} \mathrm{C}$, followed by 40 cycles of $12 \mathrm{sec}$ at $95^{\circ} \mathrm{C}$ and $40 \mathrm{sec}$ at $58.5^{\circ} \mathrm{C}$. Primers used in PCR reactions were: 5'-AATACATCAGCA CTGTTGCCTTT-3' (forward) 5'-CTCCATACATACATC TCCAAAAAGT-3' (reverse) for LINC01638 lncRNA; 5'-GAC CTCTATGCCAACACAGT-3' (forward) and 5'-AGTACTTGC GCTCAGGAGGA-3' (reverse) for $\beta$-actin. Multiple endogenous controls (such as 18S rRNA) were used and consistent results were obtained. $\mathrm{Cq}$ valus were processed using $2^{-\Delta \Delta \mathrm{Cq}}$ method (12). Expression level of LINC01638 lncRNA was normalized to the sample with the lowest expression level, which was set to 1 . All PCR products were sequenced and correct products were obtained.

ELISA. Plasma levels of TGF- $\beta 1$ were measued using Human TGF- $\beta 1$ Quantikine ELISA kit (cat. no. DB100B; R\&D Systems, Inc., Minneapolis, MN, USA). All operations were performed in strict according with manufactuer's instructions.

Cell lines and cell culture. Human PDAC cell line PL45 (ATCC ${ }^{\circledR}$ CRL-2558 ${ }^{\mathrm{TM}}$ ) and normal pancreas duct cell line
hTERT-HPNE (ATCC ${ }^{\circledR}$ CRL-4023 ${ }^{\mathrm{TM}}$ ) were purchased from the American Type Culture Collection (Manassas, VA, USA). Cell culture was performed using Dulbecco's Modified Eagle's medium (DMEM; cat. no. 30-2002; ATCC) contaning $10 \%$ fetal bovine serum (FBS; Sangon Biotech Co., Ltd.). Cells were cultured at $37^{\circ} \mathrm{C}$ with $5 \% \mathrm{CO}_{2}$. Transfection was performed using LINC01638 IncRNA expression or negative control (NC) empty vectors (pcDNA3.1), and LINC01638 lncRNA shRNA (5'-TGCTGTTGACAGTGAGCGCCTCTA GAATGTGCTACAATTATAGTGAAGCCACAGATGTATA ATTGTAGCACATTCTAGAGTTGCCTACTGCCTCGGA-3') and NC shRNA (5'-CCGGCAACAAGATGAAGAGCAC CAACTCGAGTTGGTGCTCTTCATCTTGTTGTTTTT-3') vectors (BLOCK-iT ${ }^{\mathrm{TM}}$ ) provided by GeneCopoeia, Inc. (Rockville, MD, USA). Lipofectamine ${ }^{\circledR} 3000$ reagent (Thermo Fisher Scientific, Inc.) was used to transfect vectors (10 nM) into the two cell lines $\left(5 \times 10^{4}\right.$ cells $)$. All operations were performed according to the instructions of the kit. LINC01638 lncRNA expression levels were measured by RT-qPCR at $12 \mathrm{~h}$ after transfection. Overexpression $>200 \%$ and knockdown $<50 \%$ compared with control cells (untransfected) and negative control cells (transfected with empty vectors) were achieved before subsequent experiments. For TGF- $\beta 1$ treatment experiments, cells were treated with 10, 20 and $40 \mathrm{ng} / \mathrm{ml}$ exogenous TGF- $\beta 1$ (Sigma-Aldrich; Merck KGaA, Darmstadt, Germany) at $37^{\circ} \mathrm{C}$ with $5 \% \mathrm{CO}_{2}$ for $24 \mathrm{~h}$ prior to subsequent experiments.

In vitro cell migration and invasion assay. Cells were harvested at $24 \mathrm{~h}$ following transfection and serum free cell suspensions with a cell density of $4 \times 10^{4}$ cells $/ \mathrm{ml}$ were prepared. Cell migration and invasion abilities were evaluated using Transwell migration and invasion assays. For the migration assay, the upper chamber was filled with $4 \times 10^{3}$ cells in $0.1 \mathrm{ml}$ cell suspension (prepared using DMEM without serum). The lower chamber was filled with RPMI-1640 medium (Thermo Fisher Scientific, Inc.) supplemented with 20\% FBS (Sigma-Aldrich; Merck KGaA). The chamber was maintained at $37^{\circ} \mathrm{C}$ for $12 \mathrm{~h}$ and the membranes were collected and stained with $0.5 \%$ crystal violet (Sigma-Aldrich; Merck $\mathrm{KGaA}$ ) for $20 \mathrm{~min}$ at room temperature. The same protocol was used for the invasion assay, with Matrigel (cat. no. 356234; EMD Millipore, Billerica, MA, USA) used to coat the upper chamber prior to use. Migrating and invading cells were observed under an optical microscope (magnification, $\mathrm{x} 40$; 5 random fields per membrane).

Western blot analysis. Cell lysis buffer (cat. no. P0013K; Beyotime Institute of Biotechnology, Haimen, China) was used to extract total protein with all procedures performed in strict accordance with the instructions of the kit. Bicinchoninic acid assay was performed to measure protein concentrations. Following denaturing, protein samples (30 $\mu \mathrm{g} / \mathrm{lane})$ were subjected to SDS-PAGE on $10 \%$ gels. Gel transfer to polyvinylidene difluoride membranes was performed. Then, membranes were blocked in 5\% non-fat milk for $2 \mathrm{~h}$ at room temperature, followed by incubation with rabbit anti-human primary antibodies of TGF- $\beta 1$ (1:1,500; cat. no. ab92486; Abcam) and $\beta$-actin (1:1,500; cat. no. ab8227; Abcam) overnight at $4^{\circ} \mathrm{C}$. The following day, membranes were further incubated with 


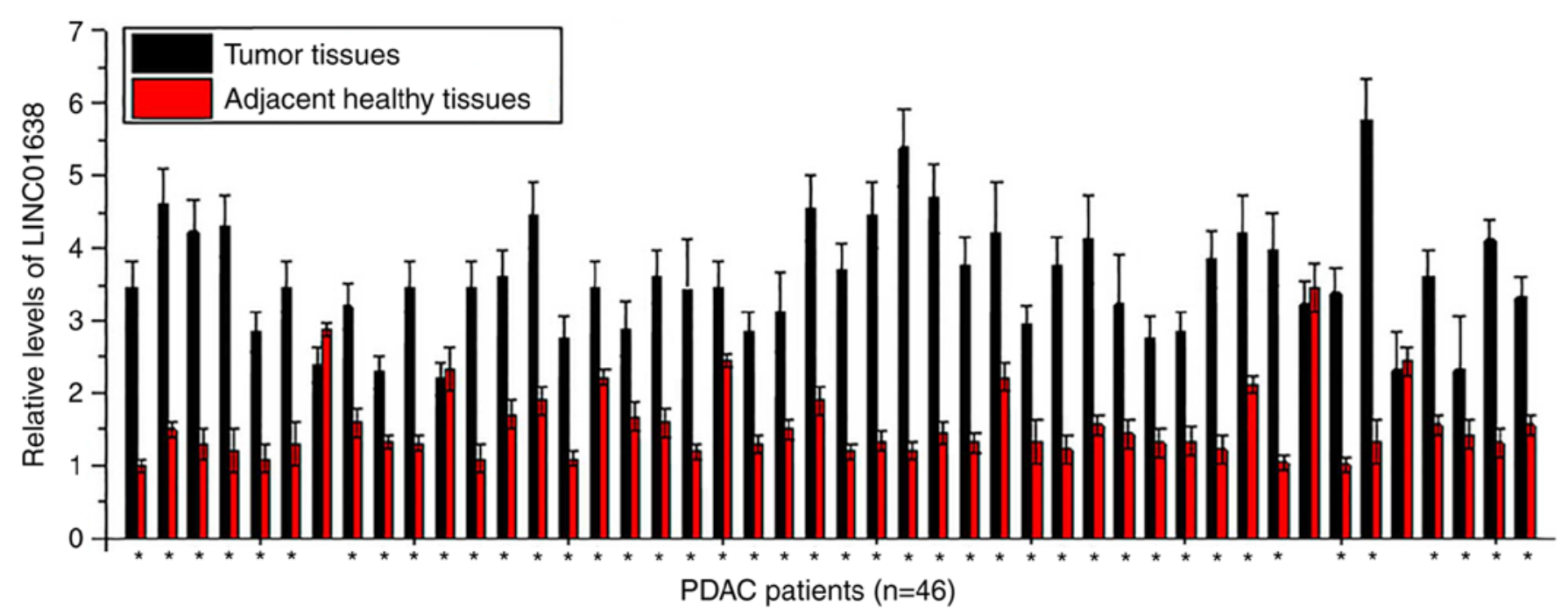

Figure 1. LINC01638 is significantly upregulated in tumor tissues than in adjacent healthy tissues in the majority of patients with PDAC ("P<0.05 tumor vs. healthy tissue). PDAC, pancreatic ductal adenocarcinoma.

Table I. Basic information of the patient and control groups.

\begin{tabular}{lcccc}
\hline & \multicolumn{2}{c}{ Sex } & & Age range \\
Groups & Male & Female & & $\begin{array}{c}\text { Average age } \\
\text { (years) }\end{array}$ \\
\hline Patient & 25 & 21 & $25-68$ & $45.5 \pm 4.9$ \\
Control & 20 & 18 & $24-69$ & $46.1 \pm 5.4$ \\
\hline
\end{tabular}

goat anti-rabbit IgG-horseradish peroxidase secondary antibody (1:1,300; cat. no. MBS435036; MyBioSource, Inc., San Diego, CA, USA) at room temperature for $2 \mathrm{~h}$. Signals were developed using ECL ${ }^{\mathrm{TM}}$ Blotting Reagents (cat. no. RPN2109; Sigma-Aldrich; Merck KGaA). Densitometry analysis was performed using Image J software (v1.6; National Institutes of Health, Bethesda, MD, USA).

Statistical analysis. GraphPad Prism 6 software (GraphPad Software, Inc., La Jolla, CA, USA) was used to process all data. All experiments were performed three times and data were presented as the mean \pm standard deviation. Data were compared by unpaired t-test (between two groups) and one-way analysis of variance followed by Tukey's test (among multiple groups). Comparisons between tumor tissues and adjacent healthy tissues were performed by paired t-test. Receiver operating characteristic (ROC) curve analysis was performed to evaluate the diagnostic value of plasma LINC01638 for PDAC, with patients with PDAC as true positive cases and healthy controls as true negative cases. Correlation between plasma levels of LINC01638 and TGF- $\beta 1$ was analyzed by Pearson correlation analysis. $\mathrm{P}<0.05$ was considered to indicated a statistically significant difference.

\section{Results}

LINC01638 is significantly upregulated in tumor tissues compared with adjacent healthy tissues in the majority of patients with PDAC. Expression of LINC01638 in tumor tissues and adjacent healthy tissues of 46 patients with PDAC was detected by RT-qPCR. As presented in Fig. 1, significantly upregulated expression levels of LINC01638 in tumor tissues than in adjacent healthy tissues were detected in 43 of 46 patients (samples from 3 sites per tissue were analyzed), indicating the potential role of LINC01638 overexpression in PDAC.

Upregulation of plasma LINC01638 distinguishes patients with PDAC from healthy controls. Plasma levels of LINC01638 in 46 patients with PDAC and 38 healthy controls were measured by RT-qPCR. As presented in Fig. 2A, the plasma levels of LINC01638 were significantly higher in patients with PDAC than in healthy controls $(\mathrm{P}<0.05)$. ROC curve analysis was performed to evaluate the diagnostic value of plasma LINC01638 for PDAC (Fig. 2B). The area under the curve was 0.8760 , with $95 \%$ confidence interval of 0.8022-0.9497 and standard error of 0.03762 $(\mathrm{P}<0.0001)$. Therefore, plasma lncRNA LINC01638 is a potential diagnostic marker for PDAC.

Plasma levels of LINC01638 and TGF- $\beta 1$ are positively correlated in patients with PDAC, but not in healthy controls. Correlations between the plasma levels of LINC01638 and various cancer-associated factors were analyzed by Pearson correlation analysis. A significant positive correlation between plasma levels of LINC01638 and TGF- $\beta 1$ was observed in patients with PDAC $\left(\mathrm{R}^{2}=0.730 ; \mathrm{P}<0.05\right.$, Fig. $\left.3 \mathrm{~A}\right)$. In addition, a significant correlation $\left(\mathrm{R}^{2}=0.1331 ; \mathrm{P}<0.05\right)$ between the plasma levels of LINC01638 and TGF- $\beta 1$ was also observed in healthy controls (Fig. 3B); however, the correlation between the two factors was notably stronger in patients with PDAC, based on the greater $\mathrm{R}^{2}$ value. Correlation between LINC01638 and other selected factors were not significant (data not shown). These data indicated the potential association between LINC01638 and TGF- $\beta 1$ in PDAC.

LINC01638 regulates TGF- $\beta 1$ expression in PDAC cells. The positive correlation between plasma levels of LINC01638 and 

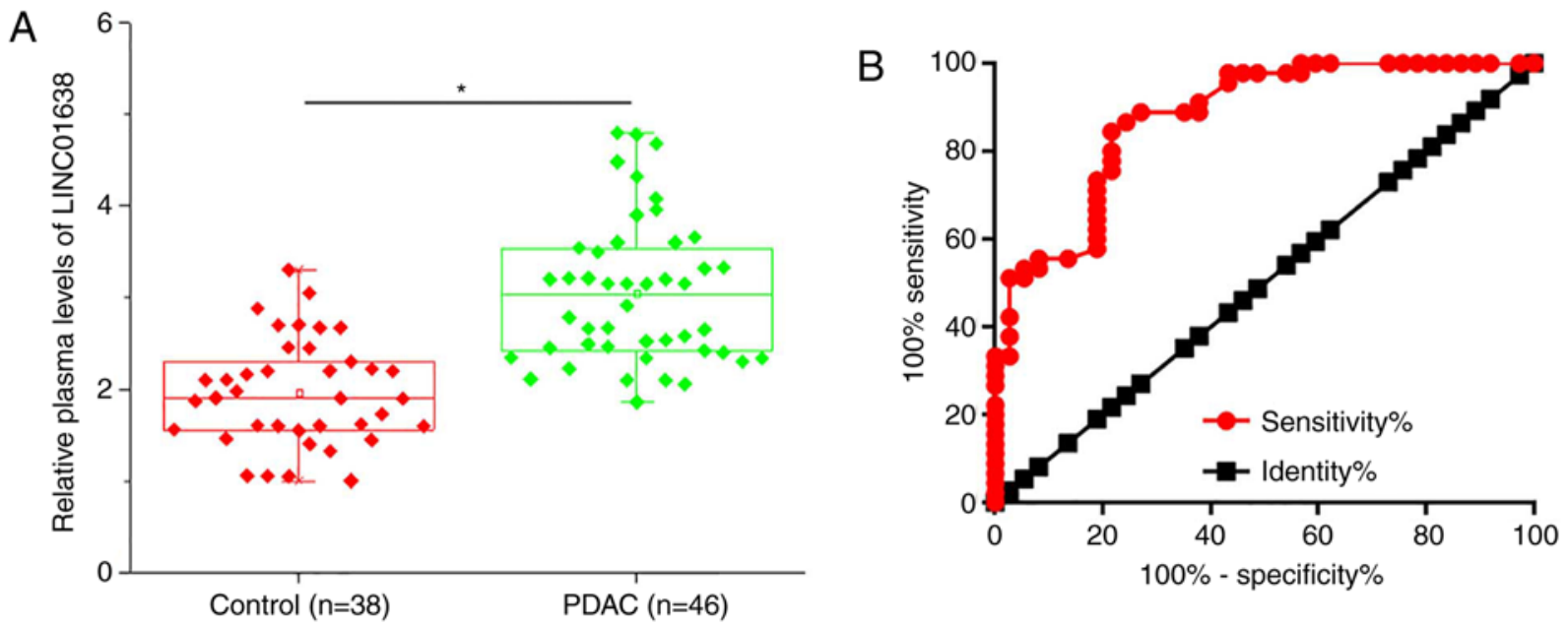

Figure 2. Upregulation of plasma LINC01638 can distinguish patients with PDAC from healthy controls. (A) Plasma levels of LINC01638 were significantly higher in PDAC patients than in healthy controls and (B) upregulation of plasma LINC01638 distinguished PDAC patients from healthy controls in receiver operating characteristic ( $\mathrm{P}<0.05)$. PDAC, pancreatic ductal adenocarcinoma.
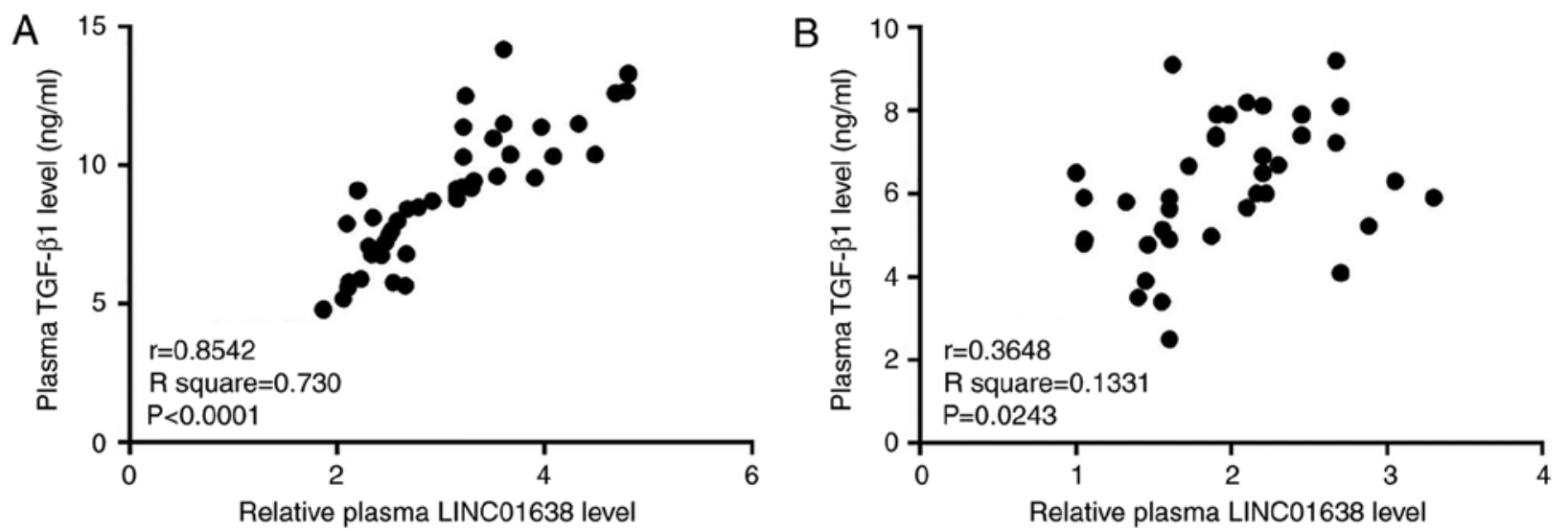

Figure 3. Plasma levels of LINC01638 and TGF- $\beta 1$ are positively correlated in patients with PDAC, and not in healthy controls. Pearson correlation analysis revealed a significant positive correlation between plasma levels of LINC01638 and TGF- $\beta 1$ in (A) patients with PDAC and (B) healthy controls. PDAC, pancreatic ductal adenocarcinoma; TGF- $\beta 1$, transforming growth factor- $\beta 1$.

TGF- $\beta 1$ in patients with PDAC indicated the potential interactions between LINC01638 and TGF- $\beta 1$. To further analyze this interaction, LINC01638 expression vectors and LINC01638 shRNA vectors were transfected into a PDAC cell line, PL45, and normal pancreas duct cell line, hTERT-HPNE. Compared with untransfected control cells and negative control cells, LINC01638 overexpression led to significantly upregulated expression of TGF- $\beta 1$ in PL45 PDAC cells (P<0.05; Fig. 4A); however, LINC01638 overexpression did not upregulate expression of TGF- $\beta 1$ in the hTERT-HPNE normal pancreas duct cell line. By contrast, LINC01638 shRNA knockdown led to significantly downregulated expression of TGF- $\beta 1$ in cells in the PDAC cell line, PL45 (P<0.05; Fig. 4B); however, LINC01638 knockdown did not alter expression of TGF- $\beta 1$ in the hTERT-HPNE normal pancreas duct cell line. In addition, treatment with exogenous TGF- $\beta 1$ at concentrations of 10,20 and $40 \mathrm{ng} / \mathrm{ml}$ did not significantly affect the expression of LINC01638 in the two cell lines (data not shown). Therefore, LINC01638 is likely to be an upstream activator of TGF- $\beta 1$ in PDAC.
LINC01638 regulates PDAC cell migration and invasion via TGF- $\beta 1$. Compared with control cells and negative control cells, LINC01638 overexpression significantly promoted migration (Fig. 5A) and invasion (Fig. 5B) of cells of the PL45 cells $(\mathrm{P}<0.05)$, but did not alter the migration of hTERT-HPNE normal pancreas duct cells. By contrast, LINC01638 shRNA knockdown inhibited migration (Fig. 5A) and invasion (Fig. 5B) of cells of PL45 PDAC cells $(\mathrm{P}<0.05)$, but did not alter the invasion of hTERT-HPNE normal pancreas duct cells. In addition, treatment with exogenous TGF- $\beta 1(10 \mathrm{ng} / \mathrm{ml})$ significantly attenuated the inhibitory effects of LINC01638 shRNA silencing on cancer cell migration (Fig. 5A) and invasion (Fig. 5B; P<0.05). Thus, LINC01638 may regulate cancer cell migration and invasion via TGF- $\beta 1$ in PDAC.

\section{Discussion}

LINC01638 is a recently identified lncRNA with a characterized oncogenic role in triple breast cancer (11). The current 

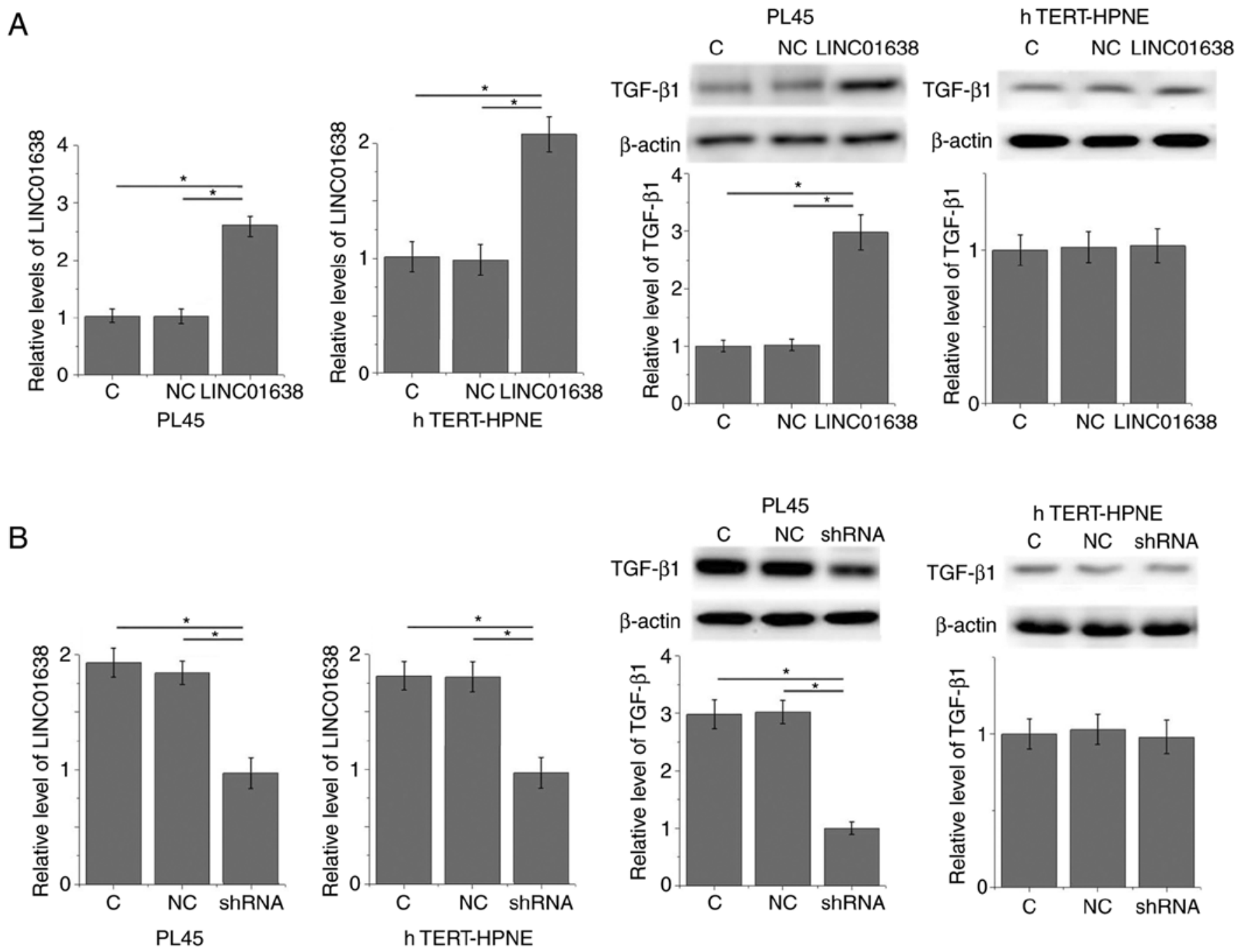

Figure 4. LINC01638 regulates TGF- $\beta 1$ expression in PDAC cells. (A) LINC01638 overexpression upregulated, (B) while LINC01638 shRNA downregulated the expression of TGF- $\beta 1$ in cells of PDAC cell line, PL45, but not in the normal pancreas duct cell line hTERT-HPNE ("P<0.05). PDAC, pancreatic ductal adenocarcinoma; C, untransfected; NC, negative control (empty vector or NC shRNA transfection); TGF- $\beta 1$, transforming growth factor- $\beta 1$; shRNA, short hairpin RNA.

study investigated the role of LINC01638 in the pathogenesis of PDAC. The findings revealed that the action of LINC01638 in PDAC is, at least partially, mediated via the activation of TGF- $\beta$ signaling.

Previous studies have demonstrated that the development and progression of PDAC is accompanied by changes in the expression pattern of numerous lncRNAs $(13,14)$. IncRNAs that are upregulated or downregulated in PDAC indicates their functionality in this disease. It has been reported that the lncRNA TMED11P is downregulated in patients with PDAC compared with healthy tissues, and the reduced expression level of lncRNA TMED11P is associated with accelerated progression and poor prognosis of this disease (15). In another study, lncRNA AFAP1-AS1 was reported to be upregulated in PDAC, which supports its role as an oncogenic lncRNA in the pathogenesis of this disease (16). Upregulation of LINC01638 has been reported in triple-negative breast cancer tissues compared with paired healthy tissue (11), while its expression pattern in other disease is unknown. In the current study, LINC01638 was upregulated in PDAC tissues compared with paired healthy tissues in the majority of patients with PDAC, indicating the role of LINC01638 as an oncogenic lncRNA in this disease.

Changes of circulating molecules provide guidance for the diagnosis of human diseases (17). Circulating lncRNAs, such as IncRNA MALAT (18), also have potential application for the diagnosis of PDAC. In the present study, plasma levels of LINC01638 were significantly higher in patients with PDAC compared with healthy controls. In effect, upregulation of plasma LINC01638 sensitively and specifically distinguished patients with PDAC from healthy controls; therefore, detecting changes in plasma LINC01638 may provide guidance for the diagnosis of PDAC. However, the expression of LINC01638 may also been affected in other human diseases, which may affect the diagnostic specificity; therefore, multiple approaches should be combined to reduce false positives.

TGF- $\beta$ is a key mediator involved in the development of multiple human diseases (19). TGF- $\beta$ interacts with multiple signaling molecules, including lncRNAs $(20,21)$; however, the crosstalk between TGF- $\beta$ signaling and lncRNAs in PDAC is not fully established. The data in 


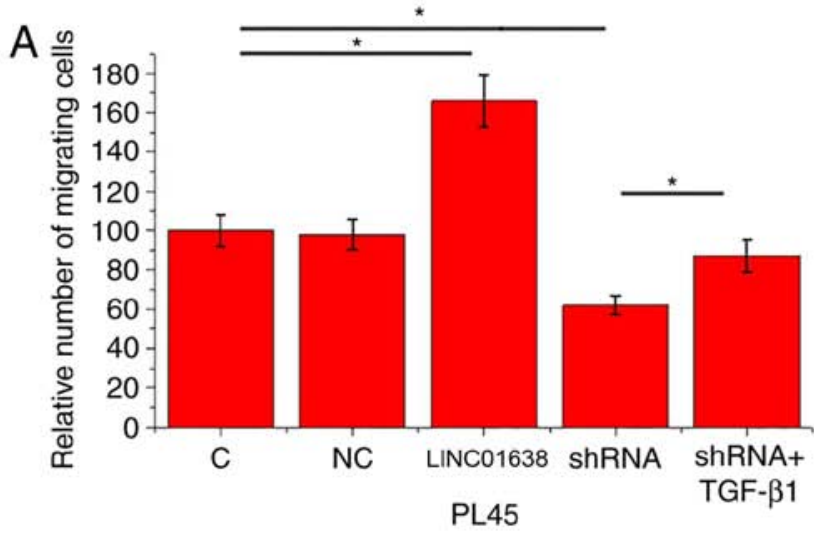

C

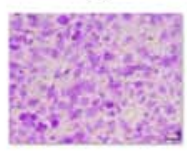

NC
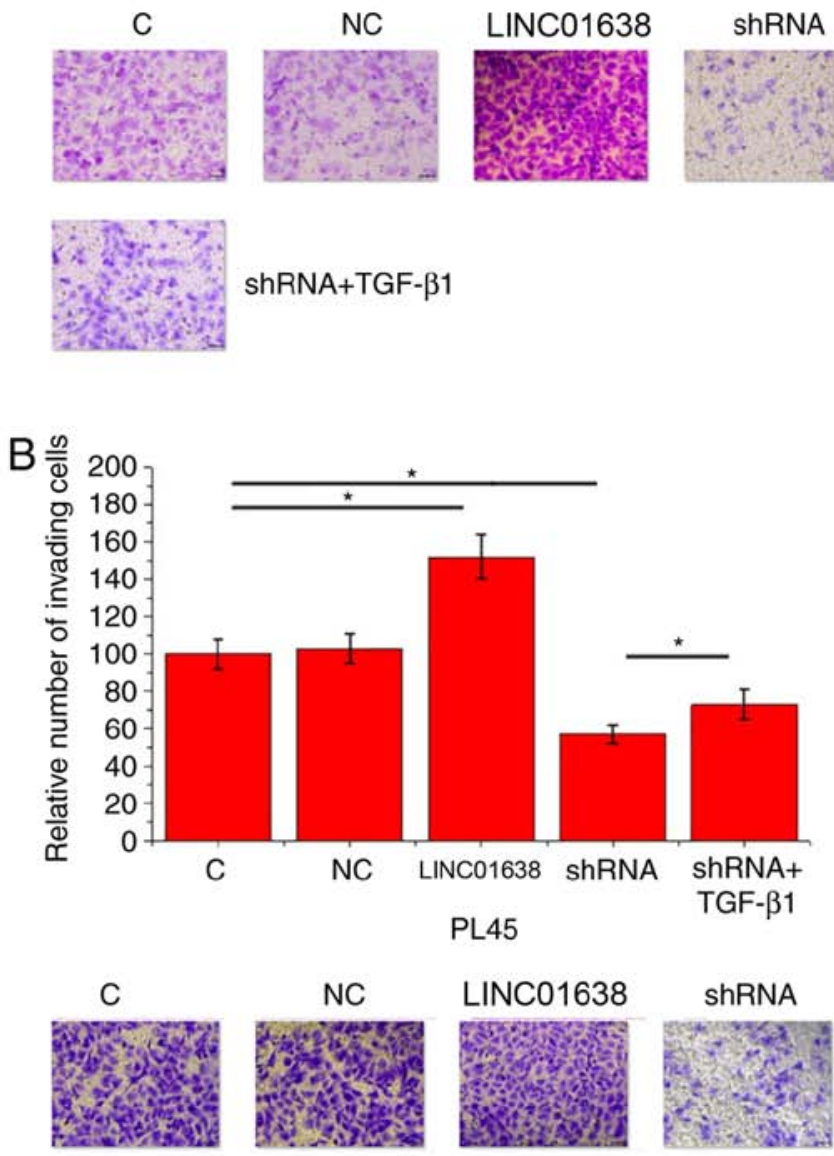
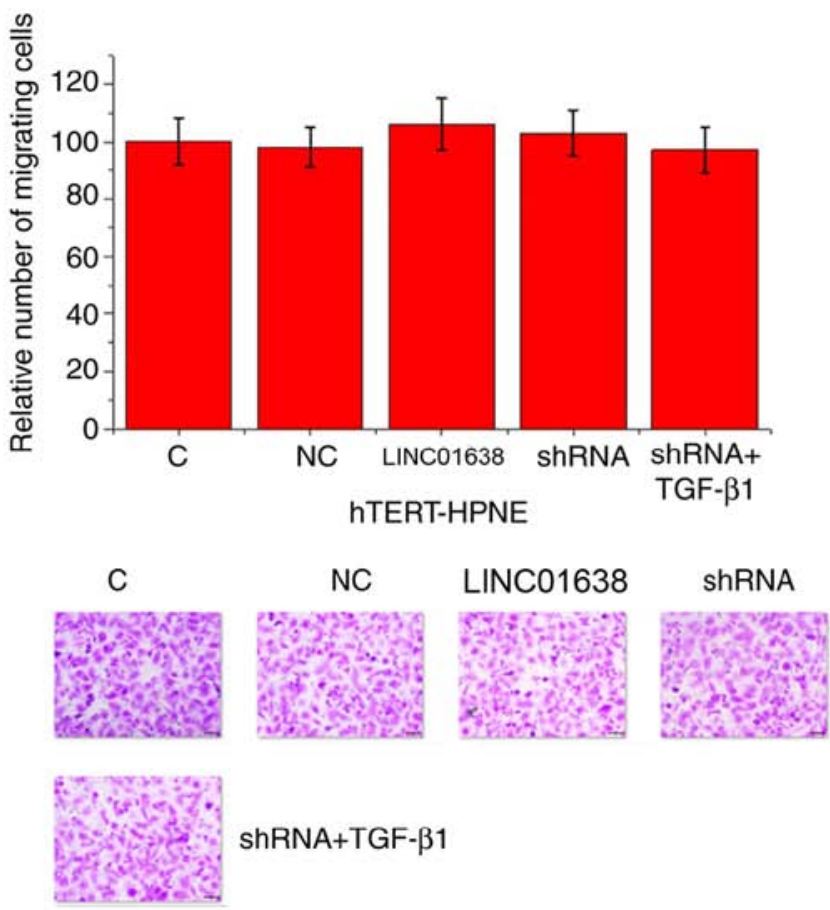

ShRNA+TGF- $\beta 1$
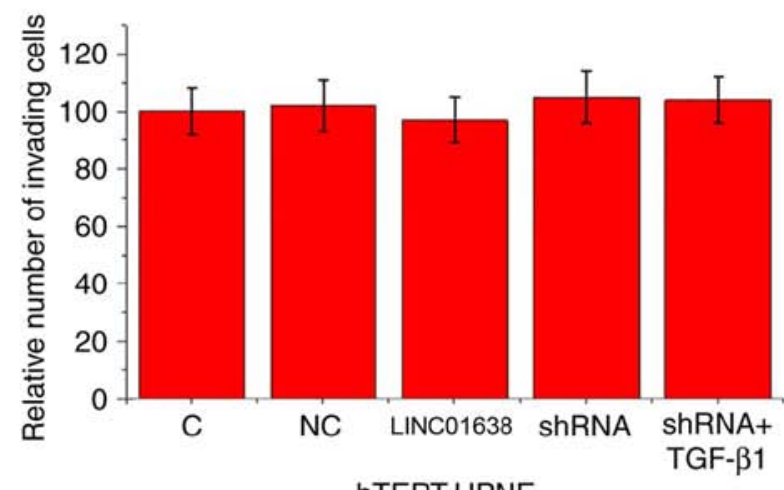

hTERT-HPNE
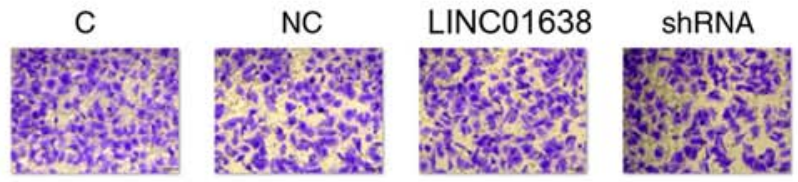

ShRNA+TGF- $\beta 1$

Figure 5. LINC01638 regulated PDAC cell migration and invasion through TGF- $\beta 1$. Effects of LINC01638 overexpression, shRNA silencing and exogenous TGF- $\beta 1$ treatment on (A) migration and (B) invasion of PL45 human PDAC cell line and hTERT-HPNE normal pancreas duct cell line. * $<0.05$. Magnification, $\mathrm{x} 40$. PDAC, pancreatic ductal adenocarcinoma; $\mathrm{C}$, untransfected; NC, negative control (empty vector transfection); shRNA, short hairpin RNA; TGF- $\beta 1$, transforming growth factor- $\beta 1$.

the present study suggest that LINC01638 may be an activator of TGF- $\beta 1$ that promotes the migration and invasion of PDAC cells. However, treatment with downstream TGF- $\beta 1$ only partially reverse the inhibitory effects of LINC01638 shRNA silencing on cancer cell migration and invasion, indicating the involvement of other pathways in LINC01638-mediated regulation of PDAC cell migration and invasion. The molecular mechanism of the interactions between TGF- $\beta 1$ and LINC01638 remains unknown. Future studies will attempt to elucidate this molecular mechanism and explore the downstream effectors of TGF- $\beta$ signaling in the regulation of EMT.

In conclusion, LINC01638 was demonstrated to be upregulated during the development of PDAC. LINC01638 is involved in the regulation of migration and invasion of PDAC cells, at least partially, via regulation of TGF- $\beta 1$. 


\section{Acknowledgements}

Not applicable.

\section{Funding}

No funding was received.

\section{Availability of data and materials}

The datasets used and/or analyzed during the current study are available from the corresponding author on reasonable request.

\section{Authors' contributions}

HL and WH designed the experiments. HL, JY and LZ performed experiments. ML, SL and DY collected and analyzed the data. WH drafted the manuscript. All authors read and approved the final manuscript.

\section{Ethics approval and consent to participate}

The present study was approved by the Ethics Committee of the West China Hospital. All patients and healthy volunteers provided written informed consent prior to their inclusion within the study.

\section{Patient consent for publication}

Not applicable.

\section{Competing interests}

The authors declare that they have no competing interests.

\section{References}

1. Siegel R, Naishadham D and Jemal A: Cancer statistics, 2013. CA Cancer J Clin 63: 11-30, 2013.

2. Rahib L, Smith BD, Aizenberg R, Rosenzweig AB, Fleshman JM and Matrisian LM: Projecting cancer incidence and deaths to 2030: The unexpected burden of thyroid, liver, and pancreas cancers in the United States. Cancer Res 74: 2913-2921, 2014.

3. Gallmeier E and Gress TM: Pancreatic ductal adenocarcinoma. Internist (Berl) 59: 805-822, 2018 (In German).

4. Ferrone CR, Pieretti-Vanmarcke R, Bloom JP, Zheng H, Szymonifka J, Wargo JA, Thayer SP, Lauwers GY, Deshpande V, Mino-Kenudson M, et al: Pancreatic ductal adenocarcinoma: Long-term survival does not equal cure. Surgery 152 (3 Suppl 1): S43-S49, 2012

5. Adamska A, Domenichini A and Falasca M: Pancreatic ductal adenocarcinoma: Current and evolving therapies. Int $\mathbf{J}$ Mol Sci 18: pii: E1338, 2017

6. Ishiwata T: Pancreatic ductal adenocarcinoma: Basic and clinical challenges for better prognosis. J Carcinog Mutagene S9: 005, 2013.
7. Principe DR, Doll JA, Bauer J, Jung B, Munshi HG, Bartholin L, Pasche B, Lee C and Grippo PJ: TGF- $\beta$ : Duality of function between tumor prevention and carcinogenesis. J Natl Cancer Inst 6: djt369, 2014.

8. Hesler RA, Huang JJ, Starr MD, Treboschi VM, Bernanke AG Nixon AB, McCall SJ, White RR and Blobe GC: TGF- $\beta$-induced stromal CYR61 promotes resistance to gemcitabine in pancreatic ductal adenocarcinoma through downregulation of the nucleoside transporters hENT1 and hCNT3. Carcinogenesis 37: 1041-1051, 2016.

9. Thakur AK, Nigri J, Lac S, Leca J, Bressy C, Berthezene P, Bartholin L, Chan P, Calvo E, Iovanna JL, et al: TAp73 loss favors Smad-independent TGF- $\beta$ signaling that drives EMT in pancreatic ductal adenocarcinoma. Cell Death Differ 23: 1358-1370, 2016.

10. Zhu Z, Xu Y, Zhao J, Liu Q, Feng W, Fan J and Wang P: miR-367 promotes epithelial-to-mesenchymal transition and invasion of pancreatic ductal adenocarcinoma cells by targeting the Smad7-TGF- $\beta$ signalling pathway. Br J Cancer 112: 1367-1375, 2015.

11. Luo L, Tang H, Ling L, Li N, Jia X, Zhang Z, Wang X, Shi L, Yin J, Qiu N, et al: LINC01638 lncRNA activates MTDH-Twist1 signaling by preventing SPOP-mediated c-Myc degradation in triple-negative breast cancer. Oncogene 37: 6166-6179, 2018.

12. Livak KJ and Schmittgen TD: Analysis of relative gene expression data using real-time quantitative PCR and the 2(-Delta Delta C(T)) method. Methods 25: 402-408, 2001.

13. Zhou Y, Gong B, Jiang ZL, Zhong S, Liu XC, Dong K, Wu HS, Yang HJ and Zhu SK: Microarray expression profile analysis of long non-coding RNAs in pancreatic ductal adenocarcinoma. Int J Oncol 48: 670-680, 2016.

14. Fu XL, Liu DJ, Yan TT, Yang JY, Yang MW, Li J, Huo YM, Liu W, Zhang JF, Hong J, et al: Analysis of long non-coding RNA expression profiles in pancreatic ductal adenocarcinoma. Sci Rep 6: 33535, 2016.

15. Zhu Z, Zhou J, Zhang Z and Li D: Decreased expression of lncRNA TMED11P is correlated with progression and prognosis in pancreatic ductal adenocarcinoma. Int J Clin Exp Pathol 9: 10550-10556, 2016.

16. Ye Y, Chen J, Zhou Y, Fu Z, Zhou Q, Wang Y, Gao W, Zheng S, Zhao X, Chen T and Chen R: High expression of AFAP1-AS1 is associated with poor survival and short-term recurrence in pancreatic ductal adenocarcinoma. J Transl Med 13: 137, 2015.

17. Poruk KE, Blackford AL, Weiss MJ, Cameron JL, He J, Goggins M, Rasheed ZA, Wolfgang CL and Wood LD: Circulating tumor cells expressing markers of tumor-initiating cells predict poor survival and cancer recurrence in patients with pancreatic ductal adenocarcinoma. Clin Cancer Res 23: 2681-2690, 2017.

18. Liu JH, Chen G, Dang YW, Li CJ and Luo DZ: Expression and prognostic significance of lncRNA MALAT1 in pancreatic cancer tissues. Asian Pac J Cancer Prev 15: 2971-2977, 2014.

19. Blobe GC, Schiemann WP and Lodish HF: Role of transforming growth factor $\beta$ in human disease. N Engl J Med 342: 1350-1358, 2000.

20. Yuan JH, Yang F, Wang F, Ma JZ, Guo YJ, Tao QF, Liu F, Pan W, Wang TT, Zhou CC, et al: A long noncoding RNA activated by TGF- $\beta$ promotes the invasion-metastasis cascade in hepatocellular carcinoma. Cancer Cell 25: 666-681, 2014.

21. Li Z, Dong M, Fan D, Hou P, Li H, Liu L, Lin C, Liu J, Su L, Wu L, et al: lncRNA ANCR down-regulation promotes TGF- $\beta$ induced EMT and metastasis in breast cancer. Oncotarget 8: 67329-67343, 2017.

This work is licensed under a Creative Commons Attribution-NonCommercial-NoDerivatives 4.0 International (CC BY-NC-ND 4.0) License. 